\title{
B-cell acute lymphoblastic leukemia in an elderly man with plasma cell myeloma and long-term exposure to thalidomide and lenalidomide: a case report and literature review
}

\author{
Ryan B. Sinit ${ }^{1}$, Dick G. Hwang ${ }^{2}$, Prakash Vishnu ${ }^{3}$, Jess F. Peterson ${ }^{4}$ and David M. Aboulafia ${ }^{1,5^{*}}$ (D)
}

\begin{abstract}
Background: The advent of the immunomodulatory imide drugs (IMiDs) lenalidomide and thalidomide for the treatment of patients with plasma cell myeloma (PCM), has contributed to more than a doubling of the overall survival of these individuals. As a result, PCM patients join survivors of other malignancies such as breast and prostate cancer with a relatively new clinical problem - second primary malignancies (SPMs) - many of which are a result of the treatment of the initial cancer. PCM patients have a statistically significant increased risk for acute myeloid leukemia (AML) and Kaposi sarcoma. IMiD treatment has also been associated with an increased risk of myelodysplastic syndrome (MDS), AML, and squamous cell carcinoma of the skin. However, within these overlapping groups, acute lymphoblastic leukemia (ALL) is much less common.

Case presentation: Herein, we describe an elderly man with PCM and a 14-year cumulative history of IMiD therapy who developed persistent pancytopenia and was diagnosed with B-cell acute lymphoblastic leukemia (B-ALL). He joins a group of 17 other patients documented in the literature who have followed a similar sequence of events starting with worsening cytopenias while on IMiD maintenance for PCM. These PCM patients were diagnosed with B-ALL after a median time of 36 months after starting IMiD therapy and at a median age of 61.5 years old.

Conclusions: PCM patients with subsequent B-ALL have a poorer prognosis than their de novo B-ALL counterparts, however, the very low prevalence rate of subsequent B-ALL and high efficacy of IMiD maintenance therapy in PCM should not alter physicians' current practice. Instead, there should be a low threshold for bone marrow biopsy for unexplained cytopenias.
\end{abstract}

Keywords: Myeloma, Lymphoblastic leukemia, Lenalidomide, Thalidomide, Therapy-related cancer

\section{Background}

Plasma cell myeloma (PCM), previously referred to as multiple myeloma, is a neoplasm of plasma cells and is the second most common hematologic malignancy [1-3]. Virtually all cases of PCM are preceded by monoclonal gammopathy of undetermined significance (MGUS). Progression from

\footnotetext{
* Correspondence: David.Aboulafia@virginiamason.org

${ }^{1}$ Floyd and Delores Jones Cancer Institute, Virginia Mason Medical Center,

1100 Ninth Avenue (C2-HEM), Seattle, WA 98101, USA

${ }^{5}$ Division of Hematology, University of Washington School of Medicine,

Seattle, WA, USA

Full list of author information is available at the end of the article
}

MGUS to smoldering (asymptomatic) PCM arises from a random second event e.g., additional genetic changes from chronic antigenic stimulation or exposure to toxins, cell cycle dysregulation, or changes in the bone marrow microenvironment $[4,5]$. Active (symptomatic) PCM is related to the expansion and infiltration of plasma cells within the bone marrow and other end-organs, resulting in significant clinical events including bone demineralization, pathologic compression fractures, cytopenias, hypercalcemia, and renal dysfunction.

(C) The Author(s). 2019 Open Access This article is distributed under the terms of the Creative Commons Attribution 4.0 International License (http://creativecommons.org/licenses/by/4.0/), which permits unrestricted use, distribution, and 
Smoldering PCM is not always treated but does require close clinical follow-up [6]. Symptomatic PCM is typically treated with induction therapy which may include combinations of corticosteroids, proteasome inhibitors, immunomodulatory imide drugs (IMiDs), DNA alkylators and, less commonly, anthracyclines and monoclonal antibodies. Eligible patients often undergo consolidation therapy with high-dose melphalan and autologous hematopoietic stem cell transplant (AHSCT) and subsequent maintenance therapy with an IMiD or a proteasome inhibitor [1, 7-9].

The advent of new therapeutic agents has dramatically improved survival for patients, particularly those who are younger than 65 years of age at diagnosis [10, 11]. Consequently, patients with PCM, like survivors of other malignancies, most notably, breast cancer, prostate cancer, Hodgkin's lymphoma (HL), and non-Hodgkin's lymphomas (NHLs), are confronted with a relatively new clinical problem-second primary malignancies (SPMs) which include both presumed treatment-related cancers and de novo second cancers. Analysis of the National Cancer Institute's (NCI) Surveillance, Epidemiology, and End Results (SEER) database of all registered cancer patients between the years 1973 and 2000 showed a 14\% increased risk of a second malignancy for these patients compared to the general population [2]. Since the increased overall survival of PCM patients is a relatively new phenomenon, the incidence of SPMs for these patients is still relatively lower than all the other cancers, reported to be $4.5 \%$ - which is lower than both HL and NHLs (both occurring at an incidence of 6.4\%). When stratified by cancer type and compared to the expected rate of malignancies in the general population, PCM patients had a statistically significant elevated risk for hematopoietic malignancies and Kaposi sarcoma. Myeloid leukemias are the most common hematologic malignancies with acute myeloid leukemia (AML) constituting $80 \%$ of all cases of leukemia following PCM [2].

As survival of PCM patients continues to increase, so too will the number of SPMs attributable to myeloma treatments. Though likely a multifactorial process, studies have associated the use of IMiDs with myelodysplastic syndrome (MDS), AML, and squamous cell carcinoma of the skin [9, 11-19]. Secondary B-cell acute lymphoblastic leukemia (B-ALL) has been reported only rarely.

Herein, we describe the case of evolving cytopenias in an elderly man with a 14-year history of IMiD treatment with recurrent skin cancers and a more recent finding of concomitant progressive PCM and B-ALL.

\section{Case presentation}

A 67-year-old Caucasian man had been in generally good health; his medical history was significant only for a resected insulinoma. In late 2003, he presented to medical attention with altered mental status and was diagnosed with pneumococcal pneumonia and bacterial meningitis. He had an elevated total protein of $12.6 \mathrm{~g} / \mathrm{dL}$ (normal, $<8.0 \mathrm{~g} / \mathrm{dL}$ ) and albumin of $3.3 \mathrm{~g} / \mathrm{dL}$ (normal, $3.4-4.8 \mathrm{~g} / \mathrm{dL}$ ). Serum protein electrophoresis (SPEP) showed a monoclonal (M) IgG kappa spike of $7.0 \mathrm{~g} / \mathrm{dL}$. Metastatic bone survey revealed multiple lytic lesions principally involving the cervical spine and right humerus. A bone marrow biopsy showed $>50 \%$ monotypic plasma cells and normal karyotype.

He began treatment for PCM in December 2003 with a regimen consisting of vincristine, doxorubicin, and dexamethasone (VAD) (Fig. 1). Despite an initial positive response, after three cycles, he had an abrupt increase in $M$ protein. The treatment was therefore changed to oral melphalan and prednisone which he continued for 5 months. $M$ protein declined significantly, but due to pancytopenia, the melphalan regimen was replaced by thalidomide and dexamethasone. He continued to take thalidomide in conjunction with dexamethasone for 36 months. Treatment was held for a month in 2006 after he sustained bilateral pathologic humeral fractures for which he received 3000 cGy of radiation therapy divided equally over 10 fractions.

While on thalidomide and dexamethasone, his PCM was well controlled, but worsening peripheral neuropathy prompted a switch to lenalidomide in November of 2007. With this change, PCM remained well controlled for the next 4 years. However, in mid-2011, lenalidomide was discontinued after he was diagnosed with a stage 1B melanoma of the right forearm which was treated with a curative wide local resection. In the 3 years prior to the melanoma diagnosis, he had also received local treatments for squamous cell cancer of the scalp and basal cell cancer over the zygomatic arch.

For 3 years after discontinuation of lenalidomide, the patient's PCM remained quiescent with an undetectable $\mathrm{M}$ protein, but during a routine follow-up evaluation in September 2014, the serum M protein increased to 1.5 $\mathrm{g} / \mathrm{dL}$, thus lenalidomide and dexamethasone were reintroduced. After 15 months, dexamethasone was discontinued to minimize the toxicity of chronic steroid therapy and he continued lenalidomide monotherapy. Over the next 2 years, he maintained an $M$ protein of $\leq 0.2 \mathrm{~g} / \mathrm{dL}$.

In 2018, at age 82, during a routine follow-up evaluation, his white blood cell (WBC) count was $0.8 \times 10^{9}$ cells/L (normal, 3.5-11.0 $\times 10^{9}$ cells/L), hematocrit $32 \%$ (normal, 39-50\%), platelet count of $89 \times 10^{9}$ cells $/ \mathrm{L}$ (normal, $150-400 \times 10^{9}$ cells/L), and a M protein concentration of $0.4 \mathrm{~g} / \mathrm{dL}$. Lenalidomide was held and when cytopenias did not improve, a posterior iliac crest bone marrow aspirate and core biopsy were obtained. Pathology findings of the marrow core biopsy showed monotypic plasma cells comprising $20 \%$ of the marrow 


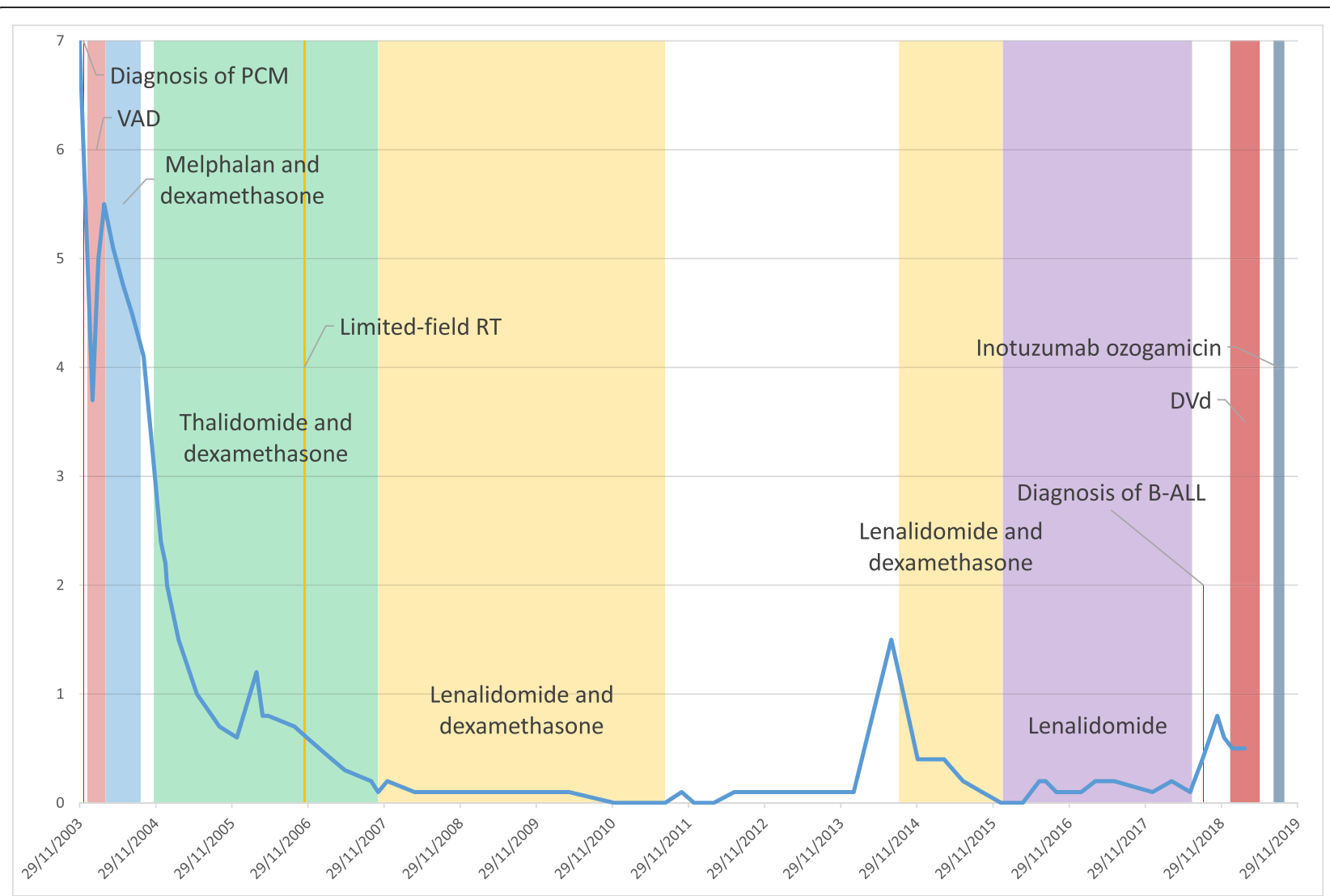

Fig. 1 Treatment of plasma cell myeloma and corresponding monoclonal protein concentration by serum electrophoresis

cellularity consistent with preexisting PCM; however, $60 \%$ of the marrow cellularity was comprised by lymphoblasts with a morphology and immunophenotype consistent with B-ALL (Fig. 2). Qualitative polymerase chain reaction was negative for the Philadelphia chromosome (BCR-ABL1 fusion). Cytogenetic analysis revealed trisomies 8 and 21 in 2 of 20 metaphases and a neartetraploid population of interphase cells including trisomies 8 and 21 (Fig. 3). Fluorescence in situ hybridization (FISH) studies for common chromosome abnormalities associated with B-ALL on interphase cells revealed a near tetraploid clone in $25 \%$ of nuclei. In addition, approximately $13 \%$ of nuclei had 3 intact copies of $M Y C$ (8q24) and RUNX1 (21q22), consistent with the trisomy 8 and trisomy 21 anomalies observed in the chromosome studies. FISH probe sets on plasma cells using immunoglobulin staining demonstrated a plasma cell clone with deletion of the TP53 gene region, trisomies 3, 7, 11, and trisomies/tetrasomies 9 and 15 .

For 6 months, the patient remained asymptomatic but with evolving red cell transfusion dependence, increasing WBC to $30 \times 10^{9}$ cells $/ \mu \mathrm{L}, 12 \%$ blasts in peripheral blood, increasing $M$ protein, and new bone lesions, he once again began palliative chemotherapy consisting of liposomal doxorubicin, vincristine, and dexamethasone (DVd) in addition to Zoledronic acid. His treatment was complicated by staphylococcal sepsis and severe and protracted pancytopenia. After recuperating, he received a second, but reduced dose of DVd which provided a good response with a resolution of blasts for a brief period of time.

At time of relapse, he began inotuzumab ozogamicin (InO), a CD22 monoclonal antibody antagonist conjugated to calicheamicin. Since initiation of $\mathrm{InO}$, there have been no circulating blasts by flow cytometric analysis 15 months from diagnosis of B-ALL. His clinical course has been complicated by low blood counts. Mprotein studies throughout his B-ALL treatment have remained stable at around $0.5 \mathrm{~g} / \mathrm{dL}$.

\section{Discussion}

PCM accounts for $1.2 \%$ of all cancers and $2 \%$ of all cancer deaths in the United States [20]. The five-year relative survival rate for PCM has increased more than two-fold since the NCI's SEER program started tracking this information; the five-year survival rate was $24.6 \%$ for those diagnosed between 1975 and 1977 compared to $52.4 \%$ of those diagnosed between 2008 and 2014. The biggest improvement in five-year survival was from 34.6 to $42.5 \%$ between 1999 


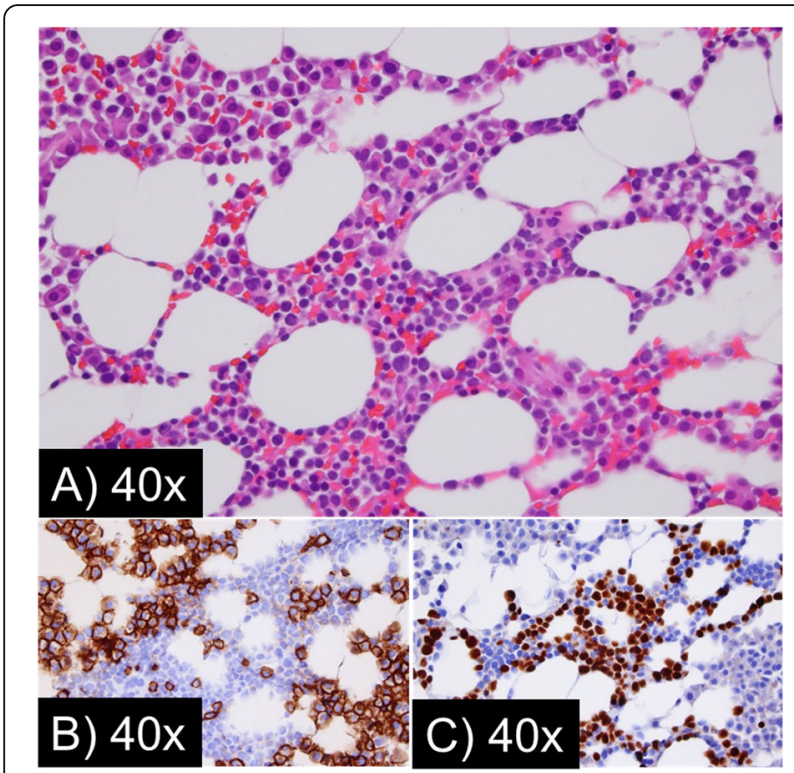

Fig. 2 Left iliac crest bone marrow aspirate from September of 2018 showing two concurrent processes: B-cell acute lymphoblastic leukemia and plasma cell myeloma. a Hematoxylin and eosin (H\&E) stain. b CD138 stain highlighting the neoplastic plasma cells of plasma cell myeloma. c Terminal deoxynucleotidyl transferase (TdT) stain highlighting the lymphoblasts of B-cell acute lymphoblastic leukemia. The three panels show the same field of view, and the two processes can also be seen on H\&E as two morphologically distinct populations

and 2001 and 2002-2004 corresponding to when thalidomide was starting to be used off-label for PCM before it was approved by the Food and Drug Administration in 2006 along with lenalidomide [20-23].

SPMs are becoming increasingly common in cancer survivors due in part to the higher survival rates of some cancers and in part to the improved treatments used to cure them [14, 24]. Most SPMs (80\%) arise in separate or independent organ systems from their primary cancers. Though not directly assessed in the SEER SPM analysis, there was an elevated risk of acute leukemias following the treatment of several cancers [2]. The etiology of any SPM is multifactorial, however increasing numbers of reviews have identified a more causal association between the treatment of primary malignancy including chemotherapy and immunosuppression, and the onset of secondary cancers [25].

The first report describing SPMs following PCM was in 1979 wherein 14 of the 364 patients (3.8\%) had developed acute leukemia following treatment of PCM with various melphalan-containing regimens $[2,13]$. Long-term studies of IMiD maintenance therapy in PCM patients have revealed a higher number of SPMs compared to patients who did not receive long-term lenalidomide. Combining data from two studies published in 2012, 48 of 537 patients (8.9\%) who were treated with lenalidomide developed a
SPM compared to 20 of 523 (3.8\%) who received placebo maintenance $[9,26]$. Several meta-analyses have also identified the significant contribution of IMiD and alkylating agent-based therapy to the risk of SPMs [18, 27-33]. In an evaluation of PCM patients who received a variety of different chemotherapy regimens, risk factors of developing MDS and subsequent AML included the use of IMiDs, older age, male gender, and a low reinfusion dose of CD34+ cells following first AHSCT [16]. A more recent analysis performed by the International Myeloma Working Group (IMWG) in 2017 also found a significantly increased incidence of SPMs following the use of lenalidomide and melphalan [12].

The mechanisms by which lenalidomide and other IMiDs contribute to SPMs are unclear. The IMiDs exert their antineoplastic effect through mechanisms such as direct cytotoxicity and indirect effects on tumor immunity [34]. Lenalidomide and thalidomide can also reactivate the lytic cycle of the Epstein-Barr virus in resting memory B-cells and lead to various lymphoproliferative disorders [35].

The vast majority of hematologic SPMs following PCM have been myeloid in nature -cases of B-ALL have been reported far more infrequently and it is unknown if the risk factors for ALL are different from those contributing to myeloid leukemias [17, 18]. To identify ALL cases following PCM, we used PubMed and the search terms "Myeloma and acute lymphocytic leukemia," "Leukemia following plasma cell myeloma," "Second primary malignancies and myeloma," and "IMiD-associated cancers." We identified an additional 17 patients in the English and non-English-language literature who were diagnosed with B-ALL following PCM (Table 1). An additional two patients had B-ALL after treatment for PCM with high-dose melphalan and AHSCT [36, 37].

Among IMiD-treated PCM patients with B-ALL, our patient is the oldest (median, 61.5 years; range, 33-82). With a cumulative exposure of 82 months, our patient is also among those with the most extensive duration of IMiD exposure (median, 35.5 months; range, 23-96), and longest latency period between starting IMiD therapy and developing ALL (median, 36 months; range, 20$179)$. Like the majority $(86 \%)$ of cases, our patient presented with worsening pancytopenia prompting a bone marrow biopsy which showed progressive PCM and a new B-ALL. Studies of the plasma cells in the aspirate were notable for a TP53 deletion and trisomies 3, 7, and 11, and trisomies/tetrasomies 9 and 15. TP53 deletion is associated with an unfavorable PCM prognosis, regardless of other abnormalities detected [38]. On interphase blast cells, FISH demonstrated a near tetraploid clone and trisomies 8 and 21; however, the prognostic significance of these findings in B-ALL is unclear [39]. Our patient is also significant in that he has had other IMiD 


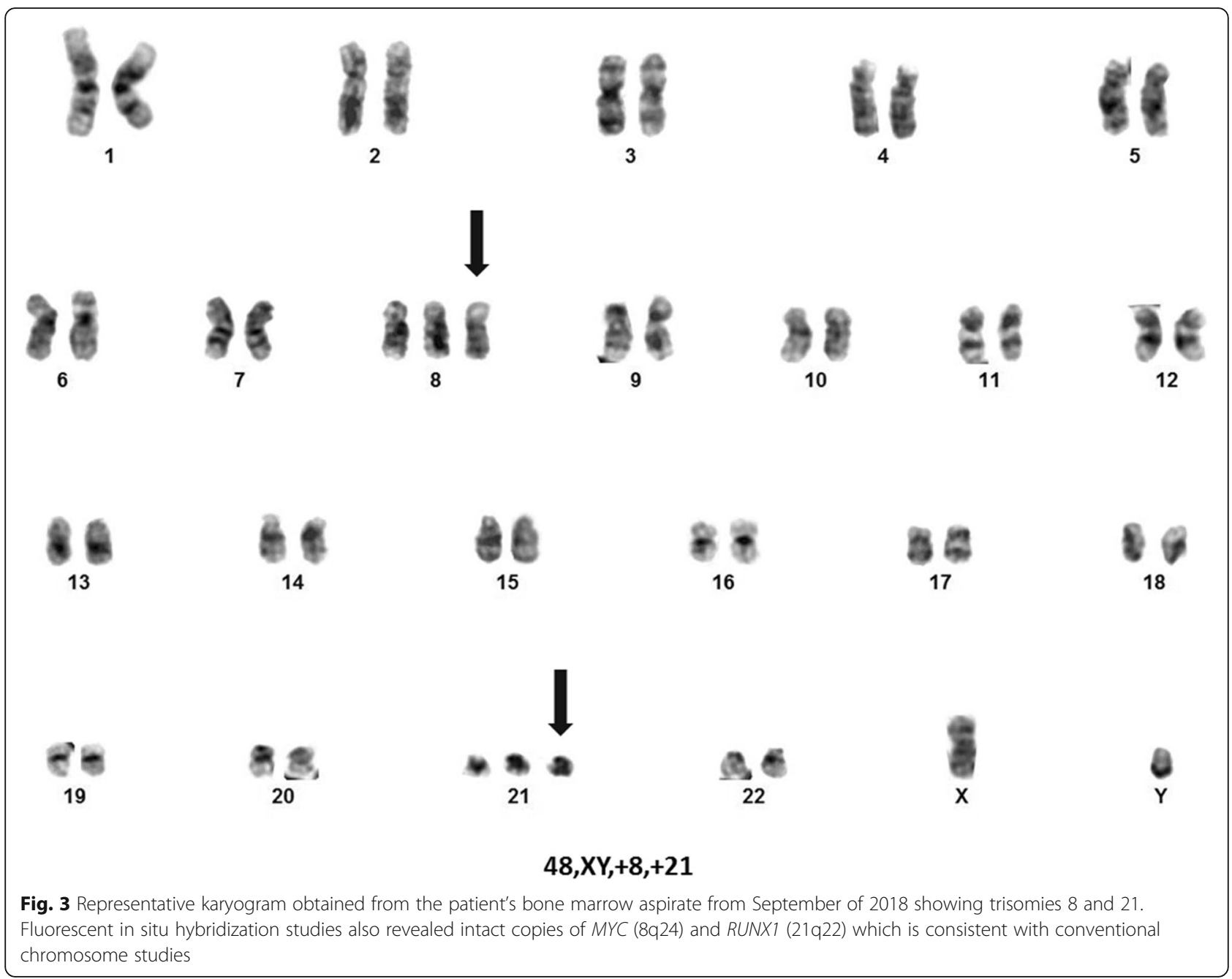

therapy-related malignancies; basal and squamous cell carcinoma of the skin and melanoma [19].

Treatment modalities for B-ALLs following PCM have varied significantly and include standard B-ALL cytotoxic chemotherapy regimens, corticosteroid monotherapy, or forgoing therapy completely (Table 1 ). In our patient, advanced age combined with evolving cytopenias precluded traditional 'induction-type' cytotoxic treatment regimens for B-ALL. We elected to use DVd as this regimen has overlapping benefits for PCM and BALL. In addition, this therapy can be delivered in the clinic without need for protracted hospitalizations. Zoledronic acid was added to minimize the risk of skeletal fractures. At time of progression, he began InO. InO was approved in 2017 and quickly integrated into the NCCN guidelines as an option for patients with relapsed/refractory ALL after showing significant improvements in progression-free survival and overall survival when compared to standard-of-care therapies $[40,41]$.

Outcomes of PCM patients with hematologic SPMs are worse than those of their de novo counterparts [24].
In an analysis of 27,000 Swedish patients with PCM and MDS or AML, survival outcomes were poorer when compared to matched patients with de novo MDS or AML - a 1.7-fold increased mortality risk was identified in the former cohort when compared to the latter. In addition, the median survival was only 2.4 months and the one-year survival rate was $16 \%$ for the former group [15]. Compared to the patients identified in our literature review, our patient was the only one to have poor cytogenetic prognostic features although many of these patients were identified at a time when FISH was not routinely performed and many of the cytogenetic aberrations identified remain of uncertain significance.

Despite the increased risk and poor prognosis of PCM patients with certain SPMs, as of 2017, the IMWG does not recommend alterations to therapeutic decision-making because the overall risk of developing a SPM is low and the therapeutic benefit of IMiD maintenance therapy significantly outweighs the risk of SPMs [12, 26, 42]. They instead recommend a low threshold for bone marrow analysis for patients with 


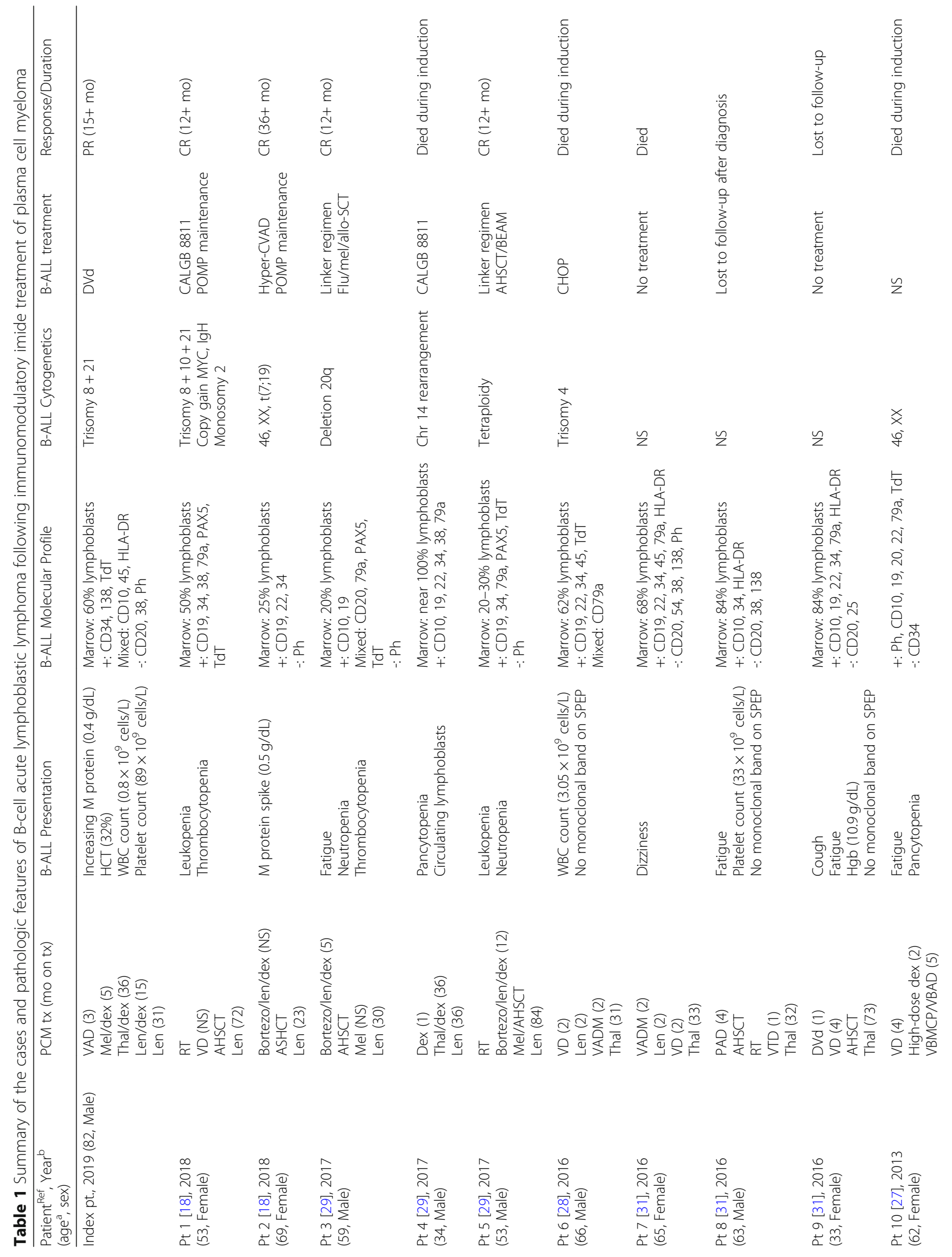


Sinit et al. BMC Cancer

(2019) 19:1147

Page 7 of 9

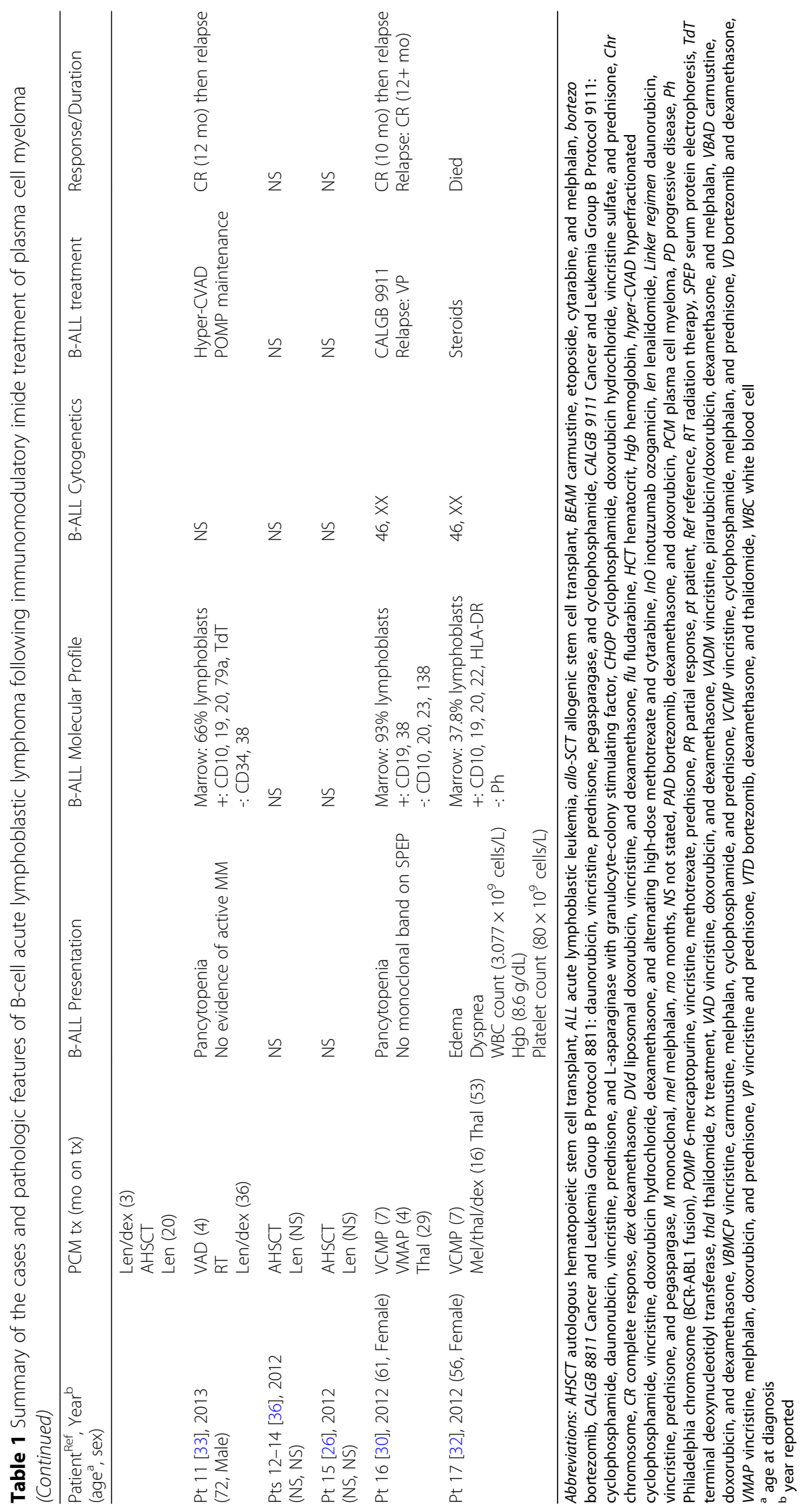


unexplained cytopenias following lenalidomide therapy withdrawal [12]. Additional research is needed to identify the weight of each risk factor, with a careful evaluation of the role of IMiDs leading to SPMs.

\section{Conclusion}

In conclusion, B-ALLs are uncommon following PCM maintenance therapy with IMiDs. Maintenance plans should not be altered since the benefits greatly outweigh the likelihood of a SPM, however there should be a low threshold to obtain a bone marrow biopsy in PCM patients on IMiD therapy who develop unexplained cytopenias. Treatment regimens for B-ALL following PCM have varied significantly and therefore more reports are required to guide decision-making in this context.

\begin{abstract}
Abbreviations
AHSCT: Autologous hematopoietic stem cell transplant; AML: Acute myeloid leukemia; B-ALL: B-Cell acute lymphoblastic leukemia; DVd: Liposomal doxorubicin, vincristine, and dexamethasone; FISH: Fluorescent in situ hybridization; HL: Hodgkin's lymphoma; IMiD: Immunomodulatory imide drug; IMWG: International Myeloma Working Group; InO: Inotuzumab ozogamicin; M protein: Monoclonal protein; MDS: Myelodysplastic syndrome; MGUS: Monoclonoal gammopathy of undetermined significance; NCl: National Cancer Institute; NHL: Non-Hodgkin's lymphoma; PCM: Plasma cell myeloma; SEER: Surveillance. Epidemiology. and End Results; SPEP: Serum protein electrophoresis; SPM: Second primary malignancy; VAD: Vincristine, doxorubicin, dexamethasone; WBC: White blood cell
\end{abstract}

\section{Acknowledgements}

We thank Virginia M. Green, PhD for her editing expertise and assistance with manuscript preparation.

\section{Authors' contributions}

RBS and DMA compiled the current case reports in the literature and wrote the manuscript. DMA and PV provided clinical decision making. DGH performed the histological examination of the bone marrow aspirate and biopsy. JFP performed the cytogenetic analysis of the bone marrow aspirate and biopsy. All authors read and approved the final manuscript.

\section{Funding}

None.

\section{Availability of data and materials}

All relevant data is contained within the manuscript. The raw data is not made available due to confidentiality.

\section{Ethics approval and consent to participate}

Not applicable.

\section{Consent for publication}

Written informed consent for publication of this case report and accompanying images was obtained from the patient. A copy of the consent is available for review by the Editor of this journal.

\section{Competing interests}

The authors declare that they have no competing interests.

\section{Author details}

${ }^{1}$ Floyd and Delores Jones Cancer Institute, Virginia Mason Medical Center, 1100 Ninth Avenue (C2-HEM), Seattle, WA 98101, USA. ²Department of Pathology, Virginia Mason Medical Center, Seattle, WA, USA. ${ }^{3}$ Department of Medical Oncology, Mayo Clinic, Jacksonville, FL, USA. ${ }^{4}$ Division of Laboratory Genetics and Genomics, Department of Laboratory Medicine and Pathology, Mayo Clinic, Rochester, MN, USA. ${ }^{5}$ Division of Hematology, University of Washington School of Medicine, Seattle, WA, USA.
Received: 9 April 2019 Accepted: 24 October 2019

Published online: 27 November 2019

\section{References}

1. Palumbo A, Anderson K. Multiple myeloma. N Engl J Med. 2011;364(11): 1046-60.

2. Curtis RE, Freedman DM, Ron E, LAG R, Hacker DG, Edwards BK, editors. New malignancies among cancer survivors: SEER Cancer Registries, 1973-2000. Bethesda: National Cancer Institute; 2006. NIH Publ. No. 05-5302

3. Swerdlow SH, Campo E, Harris NL, Jaffe ES, Pileri SA, Stein H, editors. WHO classification of Tumours of Haematopoietic and lymphoid tissues. Lyons: IARC; 2008 .

4. Kuehl WM, Bergsagel PL. Multiple myeloma: evolving genetic events and host interactions. Nat Rev Cancer. 2002;2(3):175-87.

5. Kyle RA, Therneau TM, Rajkumar SV, Offord JR, Larson DR, Plevak MF, et al. A long-term study of prognosis in monoclonal gammopathy of undetermined significance. N Engl J Med. 2002;346(8):564-9.

6. Mateos MV, Gonzalez-Calle V. Timing of treatment of smoldering myeloma: early treatment. Blood Adv. 2018:2(21):3045-9.

7. Moreau P, San Miguel J, Sonneveld P, Mateos MV, Zamagni E, Avet-Loiseau $\mathrm{H}$, et al. Multiple myeloma: ESMO Clinical Practice Guidelines for diagnosis, treatment and follow-up. Ann Oncol. 2017;28(suppl_4):iv52-61.

8. National Comprehensive Cancer Network. Multiple myeloma (Version 1.2019). NCCN Evidence Blocks ${ }^{\text {TM }}$. In: NCCN Clinical Practice Guidelines in Oncology (NCCN Guidelines ${ }^{\circledR}$ ). NCCN.org; 2018: https:/www.nccn.org/professionals/ physician_gls/pdf/myeloma_blocks.pdf. Accessed 12 Nov 2018.

9. McCarthy PL, Holstein SA, Petrucci MT, Richardson PG, Hulin C, Tosi P, et al. Lenalidomide maintenance after autologous stem-cell transplantation in newly diagnosed multiple myeloma: a meta-analysis. J Clin Oncol. 2017; 35(29):3279-89.

10. Turesson I, Velez R, Kristinsson SY, Landgren O. Patterns of improved survival in patients with multiple myeloma in the twenty-first century: a populationbased study. J Clin Oncol. 2010;28(5):830-4.

11. Kazandjian D. Multiple myeloma epidemiology and survival: A unique malignancy. Semin Oncol. 2016;43(6):676-81.

12. Musto P, Anderson KC, Attal M, Richardson PG, Badros A, Hou J, et al. Second primary malignancies in multiple myeloma: an overview and IMWG consensus. Ann Oncol. 2017;28(2):228-45.

13. Bergsagel DE, Bailey AJ, Langley GR, MacDonald RN, White DF, Miller AB. The chemotherapy on plasma-cell myeloma and the incidence of acute leukemia. N Engl J Med. 1979;301(14):743-8.

14. Thomas A, Mailankody S, Korde N, Kristinsson SY, Turesson I, Landgren O. Second malignancies after multiple myeloma: from 1960s to 2010s. Blood. 2012;119(12):2731-7.

15. Jonsdottir G, Lund SH, Bjorkholm M, Turesson I, Wahlin A, Mailankody S, et al. Survival in multiple myeloma patients who develop second malignancies: a population-based cohort study. Haematologica. 2016;101(4):e145-8.

16. Usmani SZ, Sawyer J, Rosenthal A, Cottler-Fox M, Epstein J, Yaccoby S, et al. Risk factors for MDS and acute leukemia following total therapy 2 and 3 for multiple myeloma. Blood. 2013;121(23):4753-7.

17. McCarthy PL, Holstein SA. Role of stem cell transplant and maintenance therapy in plasma cell disorders. Hematology Am Soc Hematol Educ Program. 2016;2016(1):504-11.

18. Khan AM, Muzaffar J, Murthy H, Wingard JR, Moreb JS. Acute lymphoblastic leukemia following lenalidomide maintenance for multiple myeloma: two cases with unexpected presentation and good prognostic features. Case Rep Hematol. 2018;2018:9052314.

19. Robinson AA, Wang J, Vardanyan S, Madden EK, Hebroni F, Udd KA, et al. Risk of skin cancer in multiple myeloma patients: a retrospective cohort study. Eur J Haematol. 2016;97(5):439-44.

20. Noone AM, Howlader N, Krapcho M, Miller D, Brest A, Yu M, et al., editors. SEER Cancer statistics review, 1975-2015. Bethesda: National Cancer Institute; 2018. https://seer.cancer.gov/csr/1975_2015/

21. Ghobrial IM, Rajkumar SV. Management of thalidomide toxicity. J Support Oncol. 2003;1(3):194-205.

22. U.S. Food and Drug Administration. Revlimid (lenalidomide) NDA 02-1880 Approval Letter. Approval Date: 12/27/2005. https://www.accessdata.fda. gov/drugsatfda_docs/nda/2005/021880s000_Revlimid_Approv.pdf

23. U.S. Food and Drug Administration. Thalomid (thalidomide) NDA 02-0785 Approval Letter. Approval Date: 07/16/1998. https://www.accessdata.fda. gov/drugsatfda_docs/nda/98/020785s000_Thalidomide_APPROV.pdf 
24. Patel SA. Myelodysplastic syndrome and acute myeloid leukemia risk associated with solid tumor chemotherapy. JAMA Oncol. 2018:In press. doi: https://doi.org/10.1001/jamaoncol.2018.5617.

25. Castillo JJ, Gertz MA. Secondary malignancies in patients with multiple myeloma, Waldenstrom macroglobulinemia and monoclonal gammopathy of undetermined significance. Leuk Lymphoma. 2017;58(4):773-80.

26. McCarthy PL, Owzar K, Hofmeister CC, Hurd DD, Hassoun H, Richardson PG, et al. Lenalidomide after stem-cell transplantation for multiple myeloma. $\mathrm{N}$ Engl J Med. 2012;366(19):1770-81.

27. Garcia-Munoz R, Robles-de-Castro D, Munoz-Rodriguez A, Rabasa P. Acute lymphoblastic leukemia developing during maintenance therapy with lenalidomide in a patient with multiple myeloma. Leuk Lymphoma. 2013;54(12):2753-5.

28. Li J, Zhan J, Zhang F, Ye Z, Ouyang J. Secondary lymphoblastic leukemia occurring 38 months after the primary diagnosis of multiple myeloma: a case report. Oncol Lett. 2016:12(2):847-56.

29. Tan M, Fong R, Lo M, Young R. Lenalidomide and secondary acute lymphoblastic leukemia: a case series. Hematol Oncol. 2017;35(1):130-4.

30. Tsukada Y, Hattori Y, Nakajima H, Yokoyama K, Murata M, Shimizu N, et al. B-cell acute lymphoblastic leukemia developed 5 years after autologous stem cell transplantation for multiple myeloma. Rinsho Ketsueki. 2012;53(2):219-23.

31. Junxun L, Junru L, Meilan C, Chujia L, Shaogian C, Jieyu Z, et al. Three patients with multiple myeloma developing secondary lymphoblastic leukemia: case reports and review of the literature. Tumori J. 2016; 102(Suppl. 2):S131-6.

32. Piszcz J, Bolkun L, Cichocka E, Kloczko J. Secondary acute lymphoblastic leukaemia in a multiple myeloma patient. Contemp Oncol (Pozn). 2012; 16(6):593-5.

33. Gonzalez MM, Kidd L, Quesada J, Nguyen N, Chen L. Acute myelofibrosis and acute lymphoblastic leukemia in an elderly patient with previously treated multiple myeloma. Ann Clin Lab Sci. 2013;43(2):176-80.

34. Kotla V, Goel S, Nischal S, Heuck C, Vivek K, Das B, et al. Mechanism of action of lenalidomide in hematological malignancies. J Hematol Oncol. 2009;2:36.

35. Jones RJ, lempridee T, Wang X, Lee HC, Mertz JE, Kenney SC, et al. Lenalidomide, thalidomide, and pomalidomide reactivate the Epstein-Barr virus lytic cycle through phosphoinositide 3-kinase signaling and ikaros expression. Clin Cancer Res. 2016;22(19):4901-12.

36. Lau LG, Tan LK, Koay ES, Liu TC. Acute lymphoblastic leukemia after tandem autologous stem cell transplantations for multiple myeloma. Leukemia. 2005;19(2):299-301.

37. Ueda K, Yamamoto G, Shinohara A, Hangaishi A, Kurokawa M. Early onset of acute lymphoblastic leukemia with MLL rearrangement after autologous stem cell transplantation for multiple myeloma. Ann Hematol. 2009;88(8): 813-4.

38. Gertz MA, Lacy MQ, Dispenzieri A, Greipp PR, Litzow MR, Henderson KJ, et al. Clinical implications of t $(11 ; 14)(q 13 ; q 32), t(4 ; 14)(p 16.3 ; q 32)$, and -17 p13 in myeloma patients treated with high-dose therapy. Blood. 2005; 106(8):2837-40.

39. Terwilliger T, Abdul-Hay M. Acute lymphoblastic leukemia: a comprehensive review and 2017 update. Blood Cancer J. 2017;7(6):e577.

40. Kantarjian HM, DeAngelo DJ, Stelljes M, Liedtke M, Stock W, Gökbuget N, O'Brien SM, Jabbour E, Wang T, Liang White J, Sleight B, Vandendries E, Advani AS. Inotuzumab ozogamicin versus standard of care in relapsed or refractory acute lymphoblastic leukemia: Final report and long-term survival follow-up from the randomized, phase 3 INO-VATE study. Cancer. 2019; 125(14):2474-87.

41. Kantarjian HM, DeAngelo DJ, Stelljes M, Martinelli G, Liedtke M, Stock W, Gökbuget N, O'Brien S, Wang K, Wang T, Paccagnella ML, Sleight B, Vandendries E, Advani AS. Inotuzumab Ozogamicin versus standard therapy for acute lymphoblastic leukemia. N Engl J Med. 2016;375(8):740-53.

42. Attal M, Lauwers-Cances V, Marit G, Caillot D, Moreau P, Facon T, et al. Lenalidomide maintenance after stem-cell transplantation for multiple myeloma. N Engl J Med. 2012;366(19):1782-91.

\section{Publisher's Note}

Springer Nature remains neutral with regard to jurisdictional claims in published maps and institutional affiliations.

\section{Ready to submit your research? Choose BMC and benefit from:}

- fast, convenient online submission

- thorough peer review by experienced researchers in your field

- rapid publication on acceptance

- support for research data, including large and complex data types

- gold Open Access which fosters wider collaboration and increased citations

- maximum visibility for your research: over $100 \mathrm{M}$ website views per year

At BMC, research is always in progress.

Learn more biomedcentral.com/submissions 